\title{
The Existence of Spanning Ended System on Claw-Free Graphs
}

\author{
Xiaodong Chen, Meijin Xu, and Yanjun Liu \\ College of Science, Liaoning University of Technology, Jinzhou 121001, China \\ Correspondence should be addressed to Xiaodong Chen; xiaodongchen74@126.com
}

Received 27 June 2016; Accepted 25 August 2016

Academic Editor: Xiangyu Meng

Copyright (C) 2016 Xiaodong Chen et al. This is an open access article distributed under the Creative Commons Attribution License, which permits unrestricted use, distribution, and reproduction in any medium, provided the original work is properly cited.

We prove that every connected claw-free graph $G$ contains a spanning $k$-ended system if and only if $\operatorname{cl}(G)$ contains a spanning $k$-ended system, where $\mathrm{cl}(G)$ denotes Ryjáček closure of $G$.

\section{Introduction}

Graph theory focuses on graphs composed of vertices and edges. The vertices in a graph are considered as discrete points usually discussed in control problems. Then there are a lot of results using graph theory to solve control and other application problems [1-13].

We consider only finite and simple graphs. For notation and terminology not defined here we refer to [14]. For a subgraph $H$ of a graph $G, G-H$ denotes the induced subgraph by $V(G)-V(H)$, and $G[S]$ denotes the induced subgraph by $S$ for $S \subseteq V(G)$. For $v \in V(G)$, let $N(v)$ denote the set of vertices adjacent to $v$, and $N[v]=N(v) \cup\{v\} . P[a, b]$ denotes a path with end vertices $a, b$ and a positive orientation from $a$ to $b$. For a path $P[a, b], x, y \in V(P)$, let $x P y$ denote the subpath from $x$ to $y$ with positive orientation and $y P^{-} x$ denote the subpath from $y$ to $x$ with negative orientation. Similarly, for a cycle $C$ with a given direction, we can define $C[a, b], C^{-}[a, b]$ with $a, b \in V(C)$. In the paper, we define clockwise as the positive direction of a cycle. We use $K_{m}$ to denote a complete graph with order $m$, and if $m=1$, then it is trivial vertex. A tree with at most $k$ leaves is called $k$-ended tree.

A graph is called claw-free if it does not contain $K_{1,3}$ induced subgraph. For a vertex $x \in V(G)$, let $G_{x}$ denote the graph with $V\left(G_{x}\right)=V(G)$ and $E\left(G_{x}\right)=E(G) \cup\{u v: u, v \in$ $N(x)$, and then $G_{x}$ is called the local completion of $G$ at $x$. For a graph $G, x \in V(G)$, if $G[N(x)]$ is connected, then $x$ is locally connected; if $G[N(x)]$ is a complete induced subgraph of $G$, then $x$ is simplicial; if $x$ is locally connected, but not simplicial, then $x$ is eligible.
Ryjáček [15] proposed a closure operation on a claw-free graph $G$ by joining all nonadjacent pairs of vertices in the neighbourhood of every eligible vertex till there is no eligible vertex, and then we get the $\operatorname{closure~} \mathrm{cl}(G)$. Ryjáček also gave the following result, which is considered a useful tool to research on the Hamiltonian properties of claw-free graphs.

Theorem 1 (Ryjáček [15]). If $G$ is a connected claw-free graph, then $\mathrm{cl}(G)$ is Hamiltonian if and only if $G$ is Hamiltonian.

Actually, there are a lot of results which present that $\mathrm{cl}(G)$ and $G$ have many common properties.

Theorem 2 (Brandt et al. [13]). A claw-free graph $G$ is traceable if and only if $\mathrm{cl}(G)$ is traceable.

Theorem 3 (Ryjáček et al. [16]). Let $G$ be a claw-free graph. If $\mathrm{cl}(G)$ contains 2 factors with $k$ components, then $G$ contains 2 factors with at most $k$ components.

A tree with at most $k$ leaves is called $k$-ended tree. Win [17] provided sufficient conditions for a graph to contain spanning $k$-ended trees by spanning $k$-ended system. A system of a graph which contains paths, cycles, and trivial vertices is defined by a function $f(\alpha)$ as follows:

$$
f(\alpha)= \begin{cases}1 \quad \text { if } \alpha \text { is } K_{1}, K_{2}, \text { or a cycle, } \\ 2 \quad \text { if } \alpha \text { is a path of order at least } 3\end{cases}
$$

A system $\mathcal{S}$ is called $k$-ended system if $\sum_{\alpha \in \mathcal{S}} f(\alpha) \leq k$. Moreover, if $V(\mathcal{S})=V(G)$, then $\mathcal{S}$ is called a spanning 
$k$-ended system of $G$. Obviously, if $G$ contains a spanning $k$ ended system, then $G$ contains a spanning $k$-ended tree. It follows that if a graph contains no spanning $k$-ended tree, then it contains no spanning $k$-ended systems.

In this paper, we prove that $\operatorname{cl}(G)$ can preserve the existence of spanning $k$-ended system of $G$.

Theorem 4. A claw-free graph $G$ contains a spanning $k$-ended system if and only if $\mathrm{cl}(G)$ contains a spanning $k$-ended system.

\section{Proof of Theorem 4}

We divide a $k$-ended system $\mathcal{S}$ of a graph $G$ into two sets $\mathcal{S}_{1}$ and $\mathcal{S}_{2}$ and let

$$
\begin{aligned}
& \mathcal{S}_{1}=\{\alpha \in \mathcal{S}: f(\alpha)=1\} \\
& \mathcal{S}_{2}=\{\alpha \in \mathcal{S}: f(\alpha)=2\}
\end{aligned}
$$

For every component $C \in \mathcal{S}_{1}$, we take one vertex $x_{C} \in$ $V(C)$. For every path $P \in \mathcal{S}_{2}$, let $x_{P}$ and $y_{P}$ denote the two end vertices of $P$. We define

$$
\begin{aligned}
& \text { End }\left(\mathcal{S}_{1}\right)=\bigcup_{C \in \mathcal{S}_{1}}\left\{x_{C}\right\}, \\
& \text { End }\left(\mathcal{S}_{2}\right)=\bigcup_{P \in \mathcal{S}_{2}}\left\{x_{P}, y_{P}\right\}, \\
& \operatorname{End}(\mathcal{S})=\operatorname{End}\left(\mathcal{S}_{1}\right) \cup \operatorname{End}\left(\mathcal{S}_{2}\right) .
\end{aligned}
$$

For a spanning $t$-ended system $\delta$ of a graph $G$, if there is no spanning $s$-ended system with $s<t$, then we call the system minimum spanning $t$-ended system. Obviously, for a minimum spanning $t$-ended system $\delta$ of $G, \operatorname{End}(\mathcal{S})$ is an independent set of $G$ with $|\operatorname{End}(\mathcal{S})|=t$.

In order to prove Theorem 4 , we only need to prove that the following result holds.

Theorem 5. Let $G$ be a claw-free graph with $\delta(G) \geq 2$. Then $G_{x}$ contains a spanning $k$-ended system for any vertex $x \in V(G)$ if and only if $G$ contains a spanning $k$-ended system.

Proof. Obviously, the sufficiency holds and we only need to prove the necessity. Assume $G_{x}$ contains a spanning $k$-ended system $\mathcal{S}$ which satisfies the following properties.

(T1) $\mathcal{S}$ is a minimum spanning $k$-ended system of $G_{x}$.

(T2) $E\left(G_{x}\right)-E(G)$ is minimum, subject to (T1).

(T3) $\left|\mathcal{S}_{2}\right|$ is minimum, subject to (T1) and (T2).

(T4) If $P$ contains $x$, then $P$ contains as many vertices in $N(x)$ as possible, where $P \in \mathcal{S}_{2}$ subject to (T1), (T2), and (T3).

(T5) If $C$ contains $x$, then $C$ contains as many vertices in $N(x)$ as possible, where $C \in \mathcal{S}_{1}$ subject to (T1), (T2), and (T3).

If $E(\mathcal{S})-E(G)=\emptyset$, then $\mathcal{S}$ is a spanning $k$-ended system in $G$, and we are done. Thus we assume $|E(\mathcal{S})-E(G)| \geq 1$.
Claim 1. For $P \in \mathcal{S}_{2}$, if $E(P)-E(G) \neq \emptyset$, then $x \in V(P)$.

Proof. To the contrary, suppose $x \notin V(P), P=a P b$, and $u v \in$ $E(P)-E(G)$ with $v=u^{+}$. Then $u, v \in N(x)$. Suppose $x \in c P_{1} d$, $P_{1} \in \mathcal{S}_{2}$. Since $G\left[x, u, v, x^{+}\left(x^{-}\right)\right] \neq K_{1,3}, u x^{+}\left(u x^{-}\right) \in E(G)$ or $v x^{+}\left(v x^{-}\right) \in E(G)$. Without loss of generality, suppose $x \neq d$ and $u x^{+} \in E(G)$. Then $G_{x}$ contains two paths $P_{2}=c P_{1} x v P b$ and $P_{3}=a P u x^{+} P_{1} d$. Replacing $P$ and $P_{1}$ by $P_{2}$ and $P_{3}$, then $G_{x}$ contains a spanning $k$-ended system with less edge than $E\left(G_{x}\right)-E(G)$, a contradiction to (T2).

Suppose $x \in V(C), C \in \mathcal{S}_{1}$. If $C=\{u\}$, then $G_{x}$ contains a path $P^{\prime}=a P u x v P b$ with $V\left(P^{\prime}\right)=V(C) \cup V(P)$. Replacing $P$ and $C$ by $P^{\prime}, G_{x}$ contains a spanning $(k-1)$ ended system, a contradiction to (T1). Thus $|V(C)| \geq 2$. Since $G\left[x, u, v, x^{+}\right] \neq K_{1,3}, u x^{+} \in E(G)$ or $v x^{+} \in E(G)$. Without loss of generality, suppose $u x^{+} \in E(G)$. Then $G_{x}$ contains a path $P^{\prime}=a P u C\left[x^{+}, x\right] v P b$ with $V\left(P^{\prime}\right)=V(P) \cup V(C)$. Replacing $P$ and $C$ by $P^{\prime}$, then $G_{x}$ contains a spanning $(k-1)$-ended system, a contradiction to (T1).

Claim 2. For $C \in \mathcal{S}_{1}$, if $E(C)-E(G) \neq \emptyset$, then $x \in V(C)$.

Proof. Since $E(C)-E(G) \neq \emptyset,|V(C)| \geq 2$. Suppose $u v \in$ $E(C)-E(G), v=u^{+}$. Assume to the contrary $x \notin V(C)$. Suppose $x \in a P b, P \in \mathcal{S}_{2}$. Since $G\left[x, x^{+}\left(x^{-}\right), u, v\right] \neq K_{1,3}$, $x^{+} u\left(x^{-} u\right) \in E(G)$ or $x^{+} v\left(x^{-} v\right) \in E(G)$. Without loss of generality, assume $x \neq b$ and $x^{+} u \in E(G)$. Then $G_{x}$ contains a path $P^{\prime}=b P^{-} x^{+} C^{-}[u, v] x P^{-} a$ with $V\left(P^{\prime}\right)=V(P) \cup V(C)$. Replacing $P, C$ by $P^{\prime}$, then $G_{x}$ contains a spanning $(k-1)$ ended system, a contradiction.

Suppose $x \in V\left(C^{\prime}\right), C^{\prime} \in \mathcal{S}_{1}-\{C\}$. If $V\left(C^{\prime}\right)=\{x\}$, then $G_{x}$ contains a cycle $C^{\prime \prime}=C[v, u] x v$ with $V\left(C^{\prime \prime}\right)=V(C) \cup$ $V\left(C^{\prime}\right)$. Replacing $C, C^{\prime}$ by $C^{\prime \prime}$, then $G_{x}$ contains a spanning $(k-1)$-ended system, a contradiction. If $\left|V\left(C^{\prime}\right)\right| \geq 2$, then by the preceding proof $x^{+} u \in E(G)$ or $x^{+} v \in E(G)$. Without loss of generality, assume $x^{+} u \in E(G)$. Then $G_{x}$ contains a cycle $C^{\prime \prime}=x C[v, u] C^{\prime}\left[x^{+}, x\right]$ with $V\left(C^{\prime \prime}\right)=V(C) \cup V\left(C^{\prime}\right)$. Replacing $C, C^{\prime}$ by $C^{\prime \prime}$, then $G_{x}$ contains a spanning $(k-1)$ ended system, a contradiction.

Since $\mathcal{S}$ is a disjoint system, we can get the following two results by Claims 1 and 2 .

Claim 3. For $P \in \mathcal{S}_{2}$, if $E(P)-E(G) \neq \emptyset$, then $E(\mathcal{S})-E(G) \subseteq$ $E(P)$.

Claim 4. For $C \in \mathcal{S}_{1}$, if $E(C)-E(G) \neq \emptyset$, then $E(\mathcal{S})-E(G) \subseteq$ $E(C)$.

Now we prove the case that $E(P)-E(G) \neq \emptyset$, for $P \in \mathcal{S}_{2}$. Then, by Claim $3, E(\mathcal{S})-E(G) \subseteq E(P)$. Suppose $P=a P b$, and then we can get the following results.

Claim 5. Consider the following: $|E(P)-E(G)| \leq 2$.

Proof. Suppose, to the contrary, $u_{1} v_{1}, u_{2} v_{2}, u_{3} v_{3} \in E(P)-$ $E(G)$, where $u_{1}, v_{1}, u_{2}, v_{2}, u_{3}$, and $v_{3}$ are labeled in order along the positive orientation of $P$. Since $G\left[x, u_{1}, v_{1}, v_{2}\right] \neq$ $K_{1,3}, u_{1} v_{2} \in E(G)$ or $v_{1} v_{2} \in E(G)$. Without loss of generality, assume $u_{1} v_{2} \in E(G)$. Then $G_{x}$ contains a path $P^{\prime}=a P u_{1} v_{2} P u_{3} v_{1} P u_{2} v_{3} P b$ with $V\left(P^{\prime}\right)=V(P)$ and $\mid E\left(P^{\prime}\right)-$ $E(G)|<| E(P)-E(G) \mid$, a contradiction to (T2). 
Claim 6. Consider the following: $|E(P)-E(G)|=1$.

Proof. Suppose, to the contrary, $|E(P)-E(G)|=2$ by Claim 5 and $u_{1} v_{1}, u_{2} v_{2} \in E(P)-E(G)$, where $u_{1}, v_{1}, u_{2}$, and $v_{2}$ are labeled in order along the positive orientation of $P$. By the proof of Claim 5, $u_{1} v_{2}, v_{1} u_{2} \in E(G), v_{1} v_{2} \notin E(G)$. By Claim 1, $x \in V(P)$. Assume $x \in P\left[a, u_{1}\right]$. Then $x^{+} v_{1} \notin E(G)$; otherwise $G_{x}$ contains a path $P^{\prime}=a P x u_{2} P^{-} v_{1} x^{+} P u_{1} v_{2} P b$ with $V(P)=V\left(P^{\prime}\right)$ and $\left|E\left(P^{\prime}\right)-E(G)\right|=1$, a contradiction to (T2) by Claim 3. If $x^{+} v_{2} \in E(G)$, then $G_{x}$ contains a path $P^{\prime}=a P x u_{2} P^{-} v_{1} u_{1} P^{-} x^{+} v_{2} P b$ with $V(P)=V\left(P^{\prime}\right)$ and $\mid E\left(P^{\prime}\right)-$ $E(G)|<| E(P)-E(G) \mid$, a contradiction to (T2) by Claim 3. Thus $G\left[x, x^{+}, v_{1}, v_{2}\right]=K_{1,3}$, a contradiction. By similar proof, we can prove that Claim 6 holds if $x \in P\left[v_{1}, u_{2}\right] \cup P\left[v_{2}, b\right]$.

By Claim 6, we assume that $E(P)-E(G)=\left\{u_{1} v_{1}\right\}$, where $u_{1}, v_{1}$ are labeled in order along the positive orientation of $P$, and without loss of generality assume $x \in P\left[a, u_{1}\right]$. Since $x$ is eligible, there exists at least one path in $N(x)$ connecting $u_{1}$ and $v_{1}$. Suppose $P_{0}$ is the shortest path in $N(x)$ connecting $u_{1}$ and $v_{1}$. Since $G$ is claw-free, $3 \leq\left|V\left(P_{0}\right)\right| \leq 4$. Assume $y \in V\left(P_{0}\right), u_{1} y \in E(G)$.

Claim 7. Consider the following: $y \in V(P)$.

Proof. To the contrary, suppose $y \in V\left(P^{\prime}\right)$, where $P^{\prime}=$ $P^{\prime}[c, d] \in \mathcal{S}_{2}-\{P\}$. If $y=c$, then $G_{x}$ contains a path $P_{1}=a P u_{1} y P^{\prime} d$ and a path $P_{2}=v_{1} P b$. Replacing $P$ and $P^{\prime}$ by $P_{1}$ and $P_{2}$, then $G_{x}$ contains a spanning $k$-ended system with no edge in $E\left(G_{x}\right)-E(G)$, a contradiction to (T2). Similarly, $y \neq d$. Thus $y \notin\{c, d\}$. If $y^{-} v_{1} \in E(G)$, then $G_{x}$ contains a path $P_{1}=a P u_{1} y P^{\prime} d$ and a path $P_{2}=c P^{\prime} y^{-} v_{1} P b$. Replacing $P$ and $P^{\prime}$ by $P_{1}$ and $P_{2}, G_{x}$ contains a spanning $k$-ended system $\mathcal{S}^{\prime}$ with $E\left(G_{x}\right)-E(G)=\emptyset$, a contradiction to (T2). Thus $y^{-} v_{1} \notin E(G)$. Similarly, $v_{1} y^{+} \notin E(G)$. If $y^{-} y^{+} \epsilon$ $E(G)$, then $G_{x}$ contains a path $P_{1}=a P u_{1} y v_{1} P b$ and a path $P_{2}=c P^{\prime} y^{-} y^{+} P^{\prime} d$. Replacing $P$ and $P^{\prime}$ by $P_{1}$ and $P_{2}, G_{x}$ contains a spanning $k$-ended system $S^{\prime}$ with $E\left(G_{x}\right)-E(G) \subseteq$ $E\left(P_{1}\right)$ such that $P_{1}$ contains more vertices in $N(x)$ than $P$, a contradiction to (T4). Thus $y^{-} y^{+} \notin E(G) . y v_{1} \notin E(G)$; otherwise $G\left[y, v_{1}, y^{-}, y^{+}\right]=K_{1,3}$, a contradiction. $x^{+} v_{1} \notin$ $E(G)$; otherwise $G_{x}$ contains a path $P_{1}=a P x u_{1} P^{-} x^{+} v_{1} P b$ with $V(P)=V\left(P_{1}\right)$ and $E\left(P_{1}\right)-E(G)=\emptyset$, a contradiction to (T2). Then $x^{+} y \in E(G)$ by $G\left[x, x^{+}, y, v_{1}\right] \neq K_{1,3} \cdot y^{-} x^{+} \in$ $E(G)$ or $y^{+} x^{+} \in E(G)$ by $G\left[y, y^{-}, y^{+}, x^{+}\right] \neq K_{1,3}$ and $y^{-} y^{+} \notin E(G)$. If $x^{+} y^{-} \in E(G)$, then $G_{x}$ contains two paths $P_{1}=a P x v_{1} P b$ and $P_{2}=c P^{\prime} y^{-} x^{+} P u_{1} y P^{\prime} d$. Replacing $P$ and $P^{\prime}$ by $P_{1}$ and $P_{2}, G_{x}$ contains a spanning $k$-ended system with no edge in $E\left(G_{x}\right)-E(G)$, a contradiction to (T2). If $x^{+} y^{+} \in E(G), G_{x}$ contains two paths $P_{1}=a P x v_{1} P b$ and $P_{2}=$ $c P^{\prime} y u_{1} P^{-} x^{+} y^{+} P^{\prime} d$. Replacing $P$ and $P^{\prime}$ by $P_{1}$ and $P_{2}$, then $G_{x}$ contains a spanning $(k-1)$-ended system, a contradiction. Using a similar proof, we can get a contradiction if $y_{1} \in V(C)$ with $C \in \mathcal{S}_{1}$. Thus $y \in V(P)$.

By Claim 1 without loss of generality, in the following proof, assume $x \in P\left[a, u_{1}\right)$. Then $x^{+} u_{1}, x^{-} v_{1} \in E(G)$, $x^{-} x^{+}, x^{-} u_{1}, x^{+} v_{1} \notin E(G)$ since $G$ is claw-free and by (T2).

Claim 8. Consider the following: $v_{1} y \notin E(G)$.
Proof. To the contrary, suppose $v_{1} y \in E(G)$. By Claim 7, without loss of generality, assume $y \in P\left[a, u_{1}\right]$. If $y=a$, then, replacing $P$ by $P_{1}=u_{1} P^{-} y v_{1} P b, G_{x}$ contains a spanning $k$ ended system with no edge in $E\left(G_{x}\right)-E(G)$, a contradiction to (T2). Thus $y \neq a$. If $y^{-} u_{1} \in E(G)$, then, replacing $P$ by $P_{1}=a P y^{-} u_{1} P^{-} y v_{1} P b, G_{x}$ contains a spanning $k$-ended system with no edge in $E\left(G_{x}\right)-E(G)$, a contradiction to (T2). Similarly, $y^{-} y^{+} \notin E(G)$. By $G\left[y, y^{-}, y^{+}, u_{1}\right] \neq K_{1,3}$, $y^{+} u_{1} \in E(G)$. By $G\left[y, y^{-}, y^{+}, x\right] \neq K_{1,3}, y^{-} x \in E(G)$ or $y^{+} x \in E(G)$. If $y^{-} x \in E(G)$, then, replacing $P$ by $P_{1}=a P x y^{-} P^{-} x^{+} u_{1} P^{-} y v_{1} P b, G_{x}$ contains a spanning $k-$ ended system with no edge in $E\left(G_{x}\right)-E(G)$, a contradiction to (T2). Thus $y^{+} x \in E(G)$. Replacing $P$ by path $P_{1}=$ $a P x y^{+} P u x^{+} P y v P b, G_{x}$ contains a spanning $k$-ended system with no edge in $E\left(G_{x}\right)-E(G)$, a contradiction to (T2).

Claim 9. $P$ can be transformed to a path $P_{1}$ such that $V\left(P_{1}\right)=$ $V(P), v_{1} y \in E\left(P_{1}\right)$, and $E\left(G_{x}\right)-E(G)=\left\{v_{1} y\right\}$.

Proof. By Claim 7, without loss of generality, assume $y \in$ $P\left(x, u_{1}\right)$. Since $G\left[y, y^{-}, y^{+}, x\right] \neq K_{1,3}, y^{-} y^{+}, y^{-} x, y^{+} x \in$ $E(G)$. If $y^{-} y^{+} \in E(G)$, then $P_{1}=a P y^{-} y^{+} P u_{1} y v_{1} P b$. If $y^{-} x \in$ $E(G)$, then $P_{1}=a P x y^{-} P^{-} x^{+} u_{1} P^{-} y v_{1} P b$. If $y^{+} x \in E(G)$, then $P_{1}=a P x y^{+} P u_{1} x^{+} P y v_{1} P b$.

By Claim $8, v_{1} y \notin E(G)$, and then $\left|V\left(P_{0}\right)\right|=4$. Suppose $P_{0}=u_{1} y z v_{1}$. By Claim 9 , replace $P$ by $P_{1}$. By the proof of Claim 7, $z \in P_{1}$. By the proof of Claim $9, G_{x}$ contains a spanning $k$-ended system with no edge in $E\left(G_{x}\right)-E(G)$, a contradiction to (T2). It follows that Theorem 4 holds and then Theorem 5 holds.

\section{Competing Interests}

The authors declare that they have no competing interests.

\section{Acknowledgments}

The research was supported by NSFC, Tian Yuan Special Foundation 11426125, and Educational Commission of Liaoning Province L2014239.

\section{References}

[1] S. N. Daoud, "Number of spanning trees of different products of complete and complete bipartite graphs," Mathematical Problems in Engineering, vol. 2014, Article ID 965105, 23 pages, 2014.

[2] C. Contreras-Bolton, C. Rey, S. Ramos-Cossio, C. Rodríguez, F. Gatica, and V. Parada, "Automatically produced algorithms for the generalized minimum spanning tree problem," Scientific Programming, vol. 2016, Article ID 1682925, 11 pages, 2016.

[3] X.-W. Chen, J.-G. Zhang, and Y.-J. Liu, "Research on the intelligent control and simulation of automobile cruise system based on fuzzy system," Mathematical Problems in Engineering, vol. 2016, Article ID 9760653, 12 pages, 2016.

[4] G.-X. Wen, C. L. Chen, Y.-J. Liu, and Z. Liu, "Neural-networkbased adaptive leader-following consensus control for secondorder non-linear multi-agent systems," IET Control Theory \& Applications, vol. 9, no. 13, pp. 1927-1934, 2015. 
[5] Y. J. Liu, Y. Gao, S. C. Tong, and Y. M. Li, "Fuzzy approximationbased adaptive backstepping optimal control for a class of nonlinear discrete-time systems with dead-zone," IEEE Transactions on Fuzzy Systems, vol. 24, no. 1, pp. 16-28, 2016.

[6] Y. Gao and Y.-J. Liu, "Adaptive fuzzy optimal control using direct heuristic dynamic programming for chaotic discretetime system," Journal of Vibration and Control, vol. 22, no. 2, pp. 595-603, 2016.

[7] Y. J. Liu, S. C. Tong, C. L. Chen, and D. J. Li, "Neural controller design-based adaptive control for nonlinear MIMO systems with unknown hysteresis inputs," IEEE Transactions on Cybernetics, vol. 46, no. 1, pp. 9-19, 2016.

[8] Y.-J. Liu, J. Li, S.-C. Tong, and C. L. P. Chen, "Neural network control-based adaptive learning design for nonlinear systems with full-state constraints," IEEE Transactions on Neural Networks and Learning Systems, vol. 27, no. 7, pp. 1562-1571, 2016.

[9] P. Bonsma and F. Zickfeld, "Spanning trees with many leaves in graphs without diamonds and blossoms," in LATIN 2008: Theoretical Informatics, vol. 4957 of Lecture Notes in Computer Science, pp. 531-543, Springer, 2008.

[10] G. Gutin, "Out-branchings with maximal number of leaves or internal vertices: algorithmic results and open problems," Electronic Notes in Discrete Mathematics, vol. 32, pp. 75-82, 2009.

[11] H. Matsuda, K. Ozeki, and T. Yamashita, "Spanning trees with a bounded number of branch vertices in a claw-free graph," Graphs and Combinatorics, vol. 30, no. 2, pp. 429-437, 2014.

[12] J. A. Bondy and U. S. R. Murty, Graph Theory with Applications, American Elsevier, New York, NY, USA, 1976.

[13] S. Brandt, O. Favaron, and Z. Ryjáček, "Closure and stable Hamiltonian properties in claw-free graphs," Journal of Graph Theory, vol. 34, no. 1, pp. 30-41, 2000.

[14] J. A. Bondy and U. S. R. Murty, "Graph theory with applications," Pure and Applied Mathematics Journal, vol. 20, pp. 10-13, 2004.

[15] Z. Ryjáček, “On a closure concept in claw-free graphs," Journal of Combinatorial Theory, Series B, vol. 70, no. 2, pp. 217-224, 1997.

[16] Z. Ryjáček, A. Saito, and R. H. Schelp, "Closure, 2-factors, and cycle coverings in claw-free graphs," Journal of Graph Theory, vol. 32, no. 2, pp. 109-117, 1999.

[17] S. Win, "On a conjecture of Las Vergnas concerning certain spanning trees in graphs," Journal de Mathématiques Pures et Appliquées, vol. 42, pp. 21-27, 1963. 


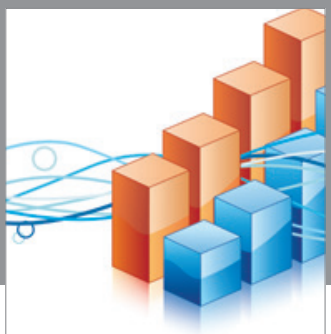

Advances in

Operations Research

vatem alat4

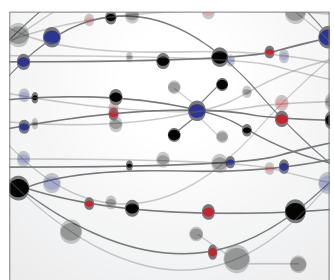

\section{The Scientific} World Journal
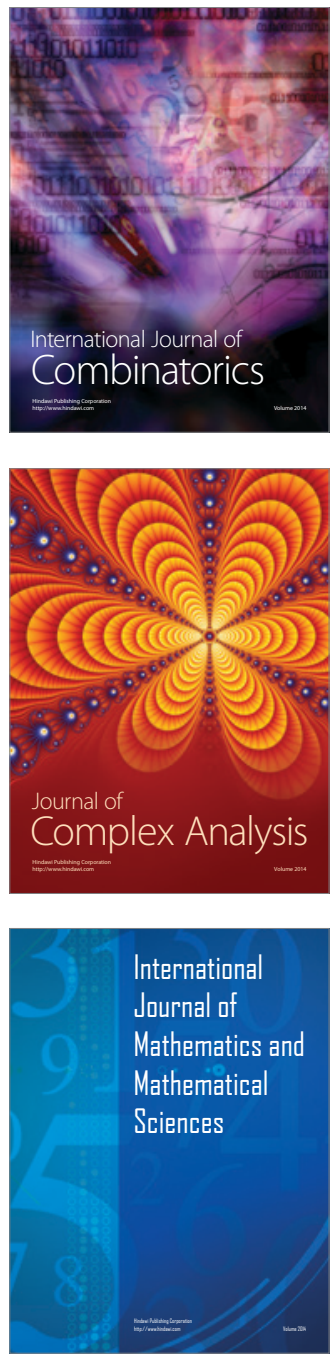
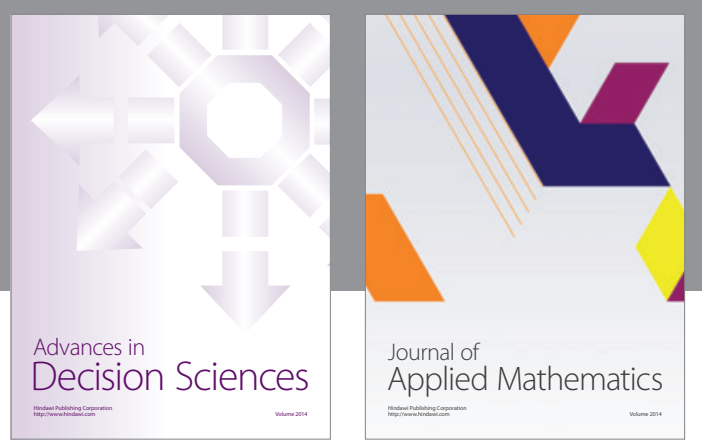

Algebra

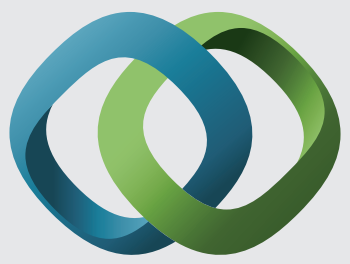

\section{Hindawi}

Submit your manuscripts at

http://www.hindawi.com
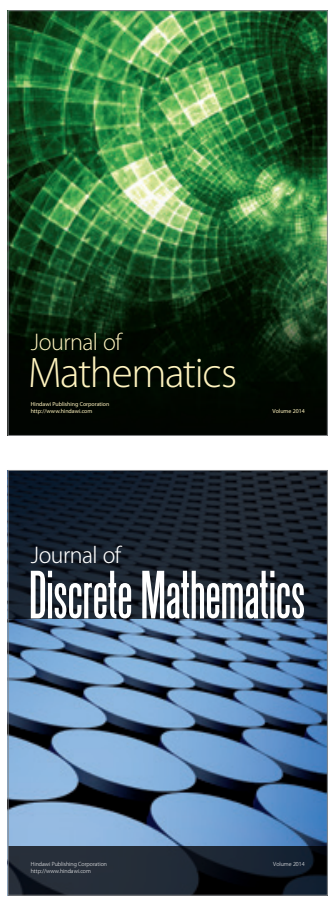

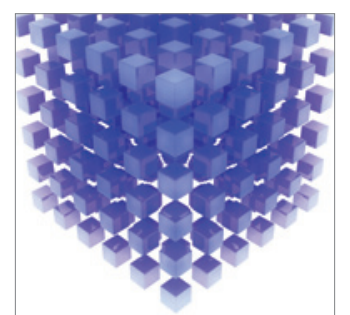

Mathematical Problems in Engineering
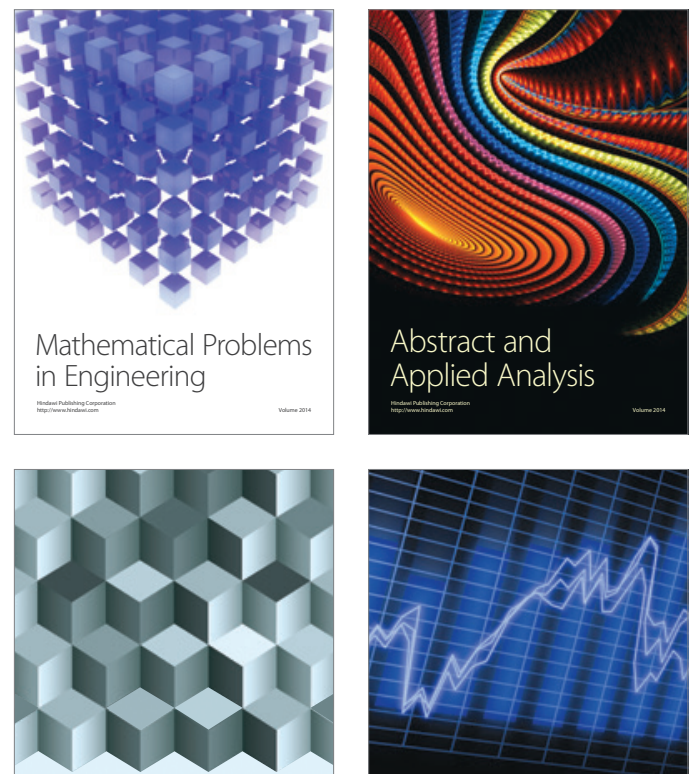

Journal of

Function Spaces

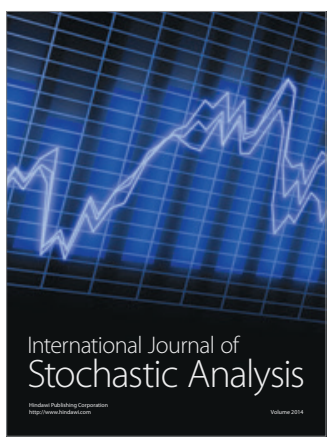

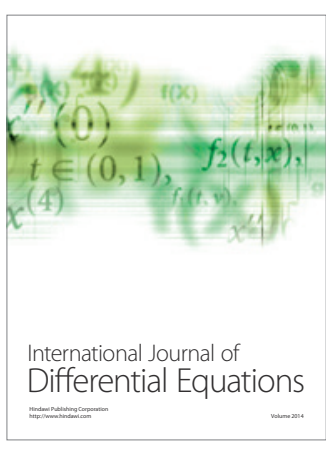
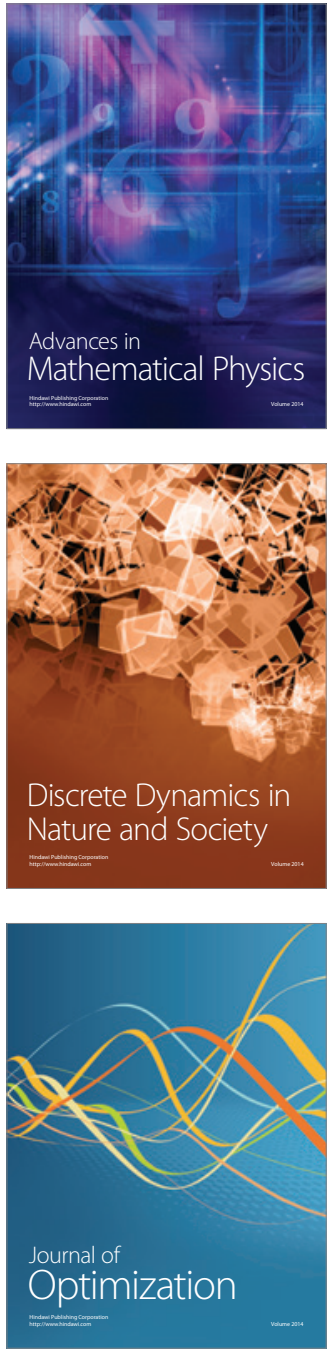